EUROPEAN ORGANIZATION FOR NUCLEAR RESEARCH

HS-RP / 035/CF

9 July 1979

HOLE COUNTING IN TRACK ETCH FOILS USING

AN IMAGE-ANALYSING COMPUTER

by

J. Dutrannois and A.H. Sullivan

Presented at the 10th International Congress on

Solid State Nuclear Track Detectors

Lyon, 2-7 July 1979 


\title{
HOLE COUNTING IN TRACK ETCH FOILS USING AN IMAGE-ANALYSING COMPUTER
}

J. Dutrannois and A.H. Sullivan

CERN, Geneva, Switzerland

\begin{abstract}
An important consideration in the use of track etch foils as the sensitive element in a neutron personnel dosimeter is the ease and reliability with which the tracks in the foils can be counted on a routine basis. An attempt has been made to adapt a microscope coupled to an image-analysing computer (Quantimet) for this purpose. A feature of the proposed hole measuring system is to use a low optical magnification and so obtain a large field of view and a large depth of focus. Under these conditions a proportion of the holes have dimensions that cannot be resolved and are detected as having an area of one or more units of the measuring grid into which the image is divided for analysis. The properties of the resulting apparent hole size distribution have been investigated from the point of view of optimizing parameters such as foil illumination, discriminator level and optical magnification as well as the apparent hole size range to be measured in order to obtain the highest sensitivity and reproducibility in the system.
\end{abstract}

\section{KEYWORDS}

Image-analysing computer, track etch foils, neutron personnel dosimeter.

\section{INTRODUCTION}

Investigations are being made into the possibility of using track etch foil (Kodak LR115) as the sensitive element in a neutron dosimeter to be used for personnel monitoring at CERN (Tymons and Tuyn, 1977, Dutrannois and Tuyn, 1977). The dosimeter itself has been described elsewhere (Dutrannois et al., 1978, Dutrannois et a1., 1979). An essential consideration in any personnel monitoring dosimeter system will be the ease and reliability with which the dosimeters can be read and the dose determined on a routine basis. As part of the study of the new dosimeter system, investigations were made into the problems associated with developing an automatic or semi-automatic track counting instrument based on a Quantimet (Cambridge Instruments model 720) image analysing computer.

METHOD

The track etch foil, after irradiation with neutrons in its cassette and subsequent development $\left(5 \frac{1}{2}\right.$ hours at $50^{\circ} \mathrm{C}$ in $2.5 \mathrm{~N} \mathrm{NaOH}$ ) shows three areas of different track 
densities, each area being about $10 \times 15 \mathrm{~mm}$. The holes in the two outer areas are mainly produced by alpha particles resulting from neutron capture in a boron irradiator. The centre area of the foil relies on the production of recoil carbon nuclei as well as spallation products from high energy neutron interactions in the foil itself and in surrounding plastic to produce observable tracks. The hole frequency is counted in these boron and plastic fields, and from the count rates obtained an estimate is made of the neutron dose equivalent to the dosimeter wearer.

To count the tracks, the foil is first mounted in a $35 \mathrm{~mm}$ slide holder which is positioned on the remotely driven $X$, $Y$ stage under the objective of an optical microscope, through which it is viewed by the vidicon scanner of the imageanalysing computer.

Maximum contrast is obtained with the microscope lamp filtered by a $5160 \AA$ narrowband interference filter, this wavelength matches well the light absorption band of the red dye of the track etch foil and is in the region of maximum sensitivity of the lead oxide cathode (plumbicon) of the video tube. The sensitivity of the scanner is adjusted from the control unit of the Quantimet which also incorporates' a "shade corrector" to correct for sensitivity differences over the area being analysed. The control unit also gives an indication of the signal level or white leve1 over the brightest $1 \%$ of the image, which can be used for setting up and checking the system gain. The video signal. passes from the control unit to the detector unit which, along with other functions, has a brightness discriminator that is used to select the lower level of image brightness that is detected before being sent to the computer-sizer units for analysis. The computer unit is set to count light areas (holes) with diameters (horizontal projection) that fall between limits set by the sizer. Seven size levels can be selected enabling six separate hole size ranges to be counted simultaneously. The settings of the sizer are in terms of light points where the standard frame image contains. $800 \times 625=5 \times 10^{5}$ such points. The Quantimet also controls the movement of the $X$, $Y$ stage of the microscope and a sequence of operations can be pre-programmed to allow automatic positioning and measuring of the foils.

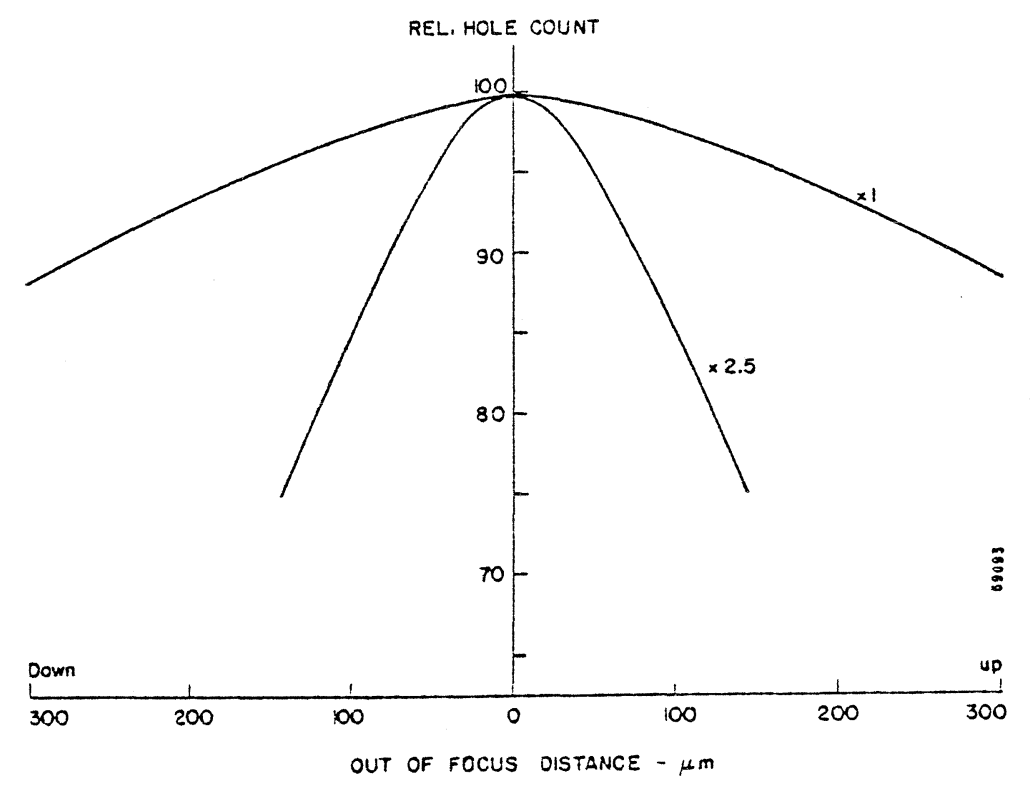

Fig. 1. Relative hole count as a function of off focus distance for the boron field of the track etch foil when using $x 1$ and $x 2.5$ objectives. 


\section{OPTIMIZATION}

Maximum sensitivity coupled to ease and speed of reading are obtained when the largest possible areas of the foils are viewed in a single field. The largest field size found to be practical was $9 \times 4 \mathrm{~mm}$, which was limited by the area of the foil available for measurement, and by the fact that the maximum step in the microscope stage is $5 \mathrm{~mm}$. This relatively large field ( $36 \mathrm{~mm}^{2}$ ) was obtained with a $\times 1$ objective together with a $\times 8$ intermediate magnification in the microscope. The low-power objective, as well as giving a wide field of view has the additional. advantage of allowing a large depth of focus, thus avoiding the necessity for automatic focusing. The initial manual focusing of the microscope is valid for all fields of all foils in a series. The dependence of track counting sensitivity on the accuracy of focus is indicated in Fig. 1, which shows the relative counts obtained when measuring tracks in a boron field of an irradiated foil as a function. of "out of focus distance" for both a $\times 2.5$ and a $x 1$ objective. Using the $x 1$ objective the foils can be out of focus by up to 250 microns before a $10 \% 10$ ss in counting efficiency occurs, whereas refocusing would become necessary after 60 microns if a $\times 2.5$ lens is used.

The dosimeter is required to measure as low doses as is possible which imposes a requirement to reduce to a minimum the background or non-radiation-induced holes that may be counted.

At the low magnification used the unit of the Quantimet measuring grid, the light point, has an associated length in the image of about 12 microns. This distance corresponds about with the minimum distance resolvable by the optical system. Apparent hole size spectra of holes under the boron irradiator after low energy neutron irradiation in the dosimeter cassette and for holes in the plastic field after irradiation with high energy neutrons are shown in Fig. 2a. More realistic hole size spectra are shown for comparison in Fig. $2 b$, where a x10 objective has been used. Both sets of spectra show a marked difference between holes induced by alpha particles from boron, and holes in the plastic field. When analysing the

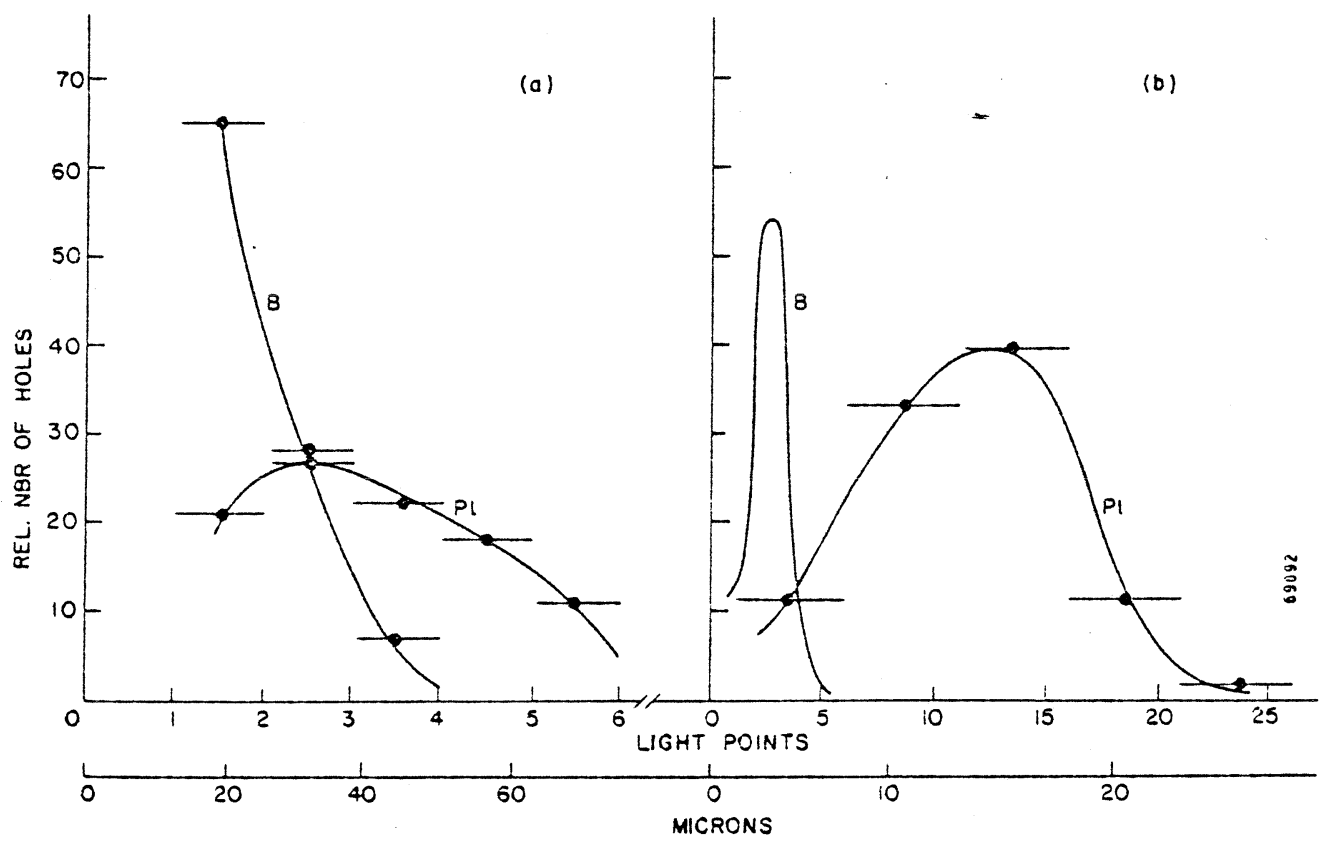

Fig. 2. Apparent hole size spectra from the boron and plastic fields of the track etch foil when using a) $x 1$ objective b) $\times 10$ objective. 
boron field the Quantimet is being used outside the limits of its resolution and is mainly observing unresolved points of light to which it will allocate a size depending on the position of the point in the measuring grid and on the uncertainty of the Quantimet projection measurement. The spectra in Fig. 2a indicate that the optimum counting window will be for holes with apparent horizontal projection in the range 1 to 6 light points.

The illumination of the foil is made as uniform as possible over the area being viewed through the microscope. Operation of the shade corrector allows for slight variations in intensity of illumination. The light intensity is adjusted such that light transmitted through the foil is just detectable above the vidicon dark current. The gain, or light sensitivity, is set such that this threshold level is at less than $10 \%$ of the brightness range that the instrument can handle. Once set up, the product of intensity of illumination and gain is fixed and is checked from time to time by viewing a $0.2 \%$ neutral grey filter in the place of the track foil, which should give a specified white level reading. It was found that gain and illumination could be varied over a reasonable range without affecting the hole counting sensitivity providing the white level was held constant. With constant white level, the contrast or ability to discriminate holes from light transmitted through the continuous foil can be adjusted with the brightness discriminator. Typical discriminator curves are shown in Fig. 3 obtained when viewing holes originating from different irradiators. "These curves have been normalized to unity at a discriminating level of 10 units on the instrument scale. No obvious optimum is indicated except that counting efficiency decreases with discriminator setting and hence the lowest discriminator level that is consistent with required contrast under all conditions will give the highest sensitivity.

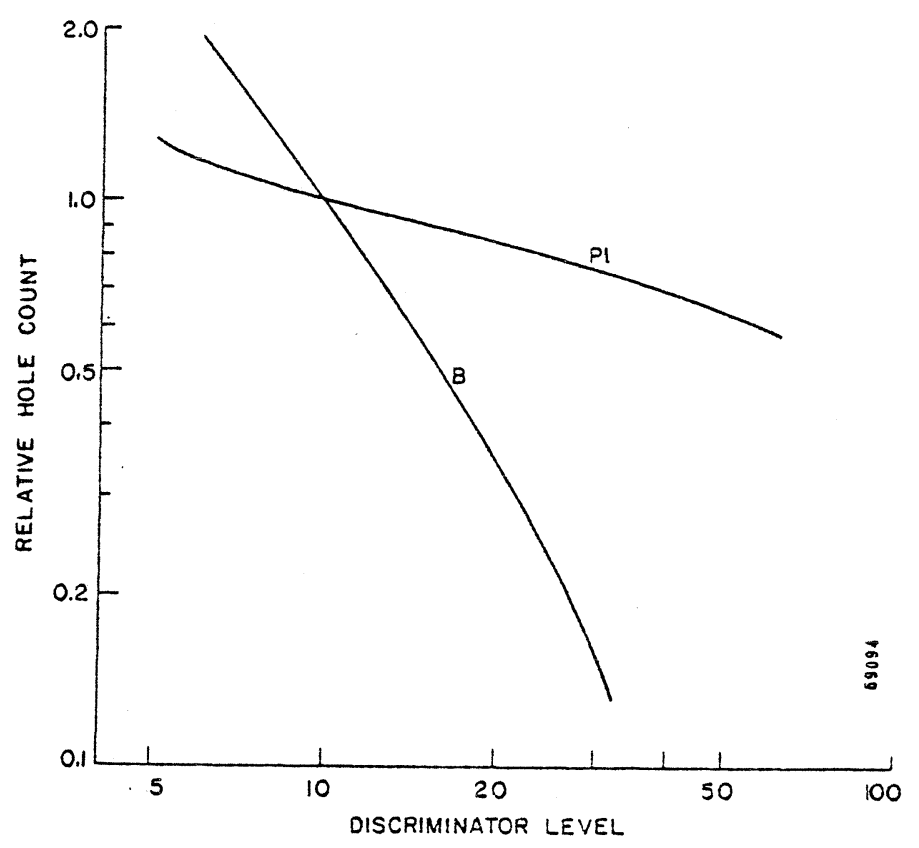

Fig. 3. Hole count as a function of brightness discriminator for holes in boron and plastic fields (x1 objective). 


\section{DISCUSSION AND CONCLUSIONS}

The basic foil measurement cycle starts with viewing an area on the first boron field where holes are counted in the size range 1 to 6 light points horizontal projection. The microscope stage then automatically moves $5 \mathrm{~mm}$ when $36 \mathrm{~mm}^{2}$ of the plastic field is counted; after a further $5 \mathrm{~mm}$ movement the plastic field is still in view when a second measurement is made. The final step brings the second boron field under the objective. Having automatically completed the cycle the stage returns to its original position under control of the Quantimet.

The problems encountered with the system relate mainly to a question of the day-to-day reproducibility of the count rate of holes induced by the boron irradiators. The count rate for these holes depends critically on the discriminator level, illumination, vidicon gain and on the reproducibility of the hole size threshold of 1 light point. The count rate was also found to be dependent on the settings of apertures in the optical system. Over a limited range changes in resolution are automatically corrected by the changes in sensitivity necessary to keep the white level constant. However, these instabilities can be corrected for by periodically counting standard foils.

More critical for the overall dosimeter system are the variations in optical thickness between developed foils, which can result in variations in hole count in the boron fields even in identically treated foils. These effects are much less critical for the holes in the plastic field, the severity of the effect being more or less in proportion to the slopes in the bias curves shown in Fig. 3 . The dependence of counting efficiency on the optical system becomes apparent when counting with different magnifications, hence hole count can only be considered relative, particularly for the very small holes that predominate in the boron field.

An overall system for automatic routine counting of track etch foils from a neutron dosimeter could be made to work, employing a large field of view and automatic scanning of the different areas of interest on the foils with the Quantimet. Although the Quantimet is being used to measure parameters outside the recommended range, its features, such as brightness and hole size range discrimination, are essential in a system required to measure the very low hole frequencies expected in the routine use of the neutron dosimeter.

\section{REFERENCES}

Tymons, B.J., and Tuyn, J.W.N. (1977). Health Physics, 32, 547.

Dutrannois, J., and Tuyn, J.W.N. (1977). Nuc1. Inst. Meth., 147, 177.

Dutrannois, J., Höfert, M., and Tuyn, J.W.N. (1978). Proc. IAEA Symp.

"Advances in Radiation Protection Monitoring", Stockholm.

Dutrannois, J., Höfert, M., Sullivan, A.H., and Tuyn, J.W.N. (1979).

"A personal neutron monitoring system based on solid state nuclear track

detectors. This Conference. 